\title{
Chemoprophylaxis in addition to mechanical prophylaxis after total knee arthroplasty surgery does not reduce the incidence of venous thromboembolism
}

\author{
Jing Loong Moses Loh ${ }^{{ }^{*}} \mathbb{D}$, Stephrene Chan ${ }^{2}$, Keng Lin Wong ${ }^{3}$, Sanjay de Mel ${ }^{4}$ and Eng Soo Yap ${ }^{4^{*}}$
}

\begin{abstract}
Background: Venous thromboembolism (VTE) of the lower limbs is an important complication post total knee arthroplasty (TKA). Current guidelines recommend routine chemical prophylaxis to all patients undergoing this procedure but this is rarely done in Asia as it is believed that Asians have a lower risk of VTE. However, recent evidence suggests otherwise.
\end{abstract}

Aims: We evaluated the incidence of DVT after TKA in a multi-ethnic Asian population with and without pharmacological prophylaxis, as well as the management and outcome of patients with post-operative DVTs.

Methods: We conducted a retrospective study of consecutive patients who underwent TKA in our hospital from 1st January 2004 to 30th December 2014. All patients were on mechanical thromboprophylaxis via calf pumps after TKA with a postoperative day 3 to 5 doppler ultrasound (DUS) of bilateral lower limbs. 2258 (80.7\%) patients did not receive additional chemoprophylaxis, while 540 (19.3\%) received chemoprophylaxis on top of mechanical thromboprophylaxis. All patients who received chemoprophylaxis were administered the drug until they were ambulating, with a median administration duration of 6 days. Patients were followed up for a period of 3 months for recurrence of DVTs and 24 months for postoperative outcome scores.

Results: Two thousand nine hundred seventy-eight patients had DUS of the lower limbs with 134 diagnosed with DVT giving an incidence of 4.5\%. Six of these patients had concurrent PEs. There were 26 (19.4\%) proximal DVTs and 108 (80. 6\%) distal DVTs. After 3 months of follow up, no additional VTE occurred. None of the DVTs or PEs progressed. All DVTs with accompanying PE were proximal. 102 out of 2200 patients (4.6\%) without chemoprophylaxis developed DVT as compared to 32 out of 540 patients (5.9\%) with chemoprophylaxis, which was not statistically significant $(p=0.13)$. 19 (0.8\%) proximal and 83 (3.8\%) distal DVT developed in the patient group without chemoprophylaxis while 4 (0.7\%) proximal and $28(5.2 \%)$ distal DVT developed in the patient group with $(p=0.62)$. Comparison of the incidence of PEs between the two groups, revealed a similar incidence with 5 out of 2200 patients (0.2\%) without chemoprophylaxis developing PE as compared to 1 out of 540 patients (0.2\%) with chemoprophylaxis ( $p=0.87$ ).

In addition, patients with chemoprophylaxis showed an association with higher post-operative outcome scores such as post op 6 months SF36 (PCS), post op 12 months SF36 (PCS), post op 12 months SF36 (MCS), post op 24 months SF36 (MCS) and post op 24 months WOMAC.

(Continued on next page)

\footnotetext{
*Correspondence: mosesloh1993@yahoo.com; Eng_Soo_YAP@nuhs.edu.sg

'Department of Orthopaedic Surgery, Singapore General Hospital, 20 College

Road, Academia, Level 4, Singapore 169865, Singapore

${ }^{4}$ Department of Hematology-Oncology, National University Cancer Institute,

National University Hospital, Singapore, Singapore

Full list of author information is available at the end of the article
}

(c) The Author(s). 2019 Open Access This article is distributed under the terms of the Creative Commons Attribution 4.0 International License (http://creativecommons.org/licenses/by/4.0/), which permits unrestricted use, distribution, and reproduction in any medium, provided you give appropriate credit to the original author(s) and the source, provide a link to the Creative Commons license, and indicate if changes were made. The Creative Commons Public Domain Dedication waiver (http://creativecommons.org/publicdomain/zero/1.0/) applies to the data made available in this article, unless otherwise stated. 
(Continued from previous page)

Conclusion: In one of the largest Asian studies specifically investigating the incidence of DVT after TKA, we found that the incidence is low at 4.5\%. This is in contrast to recent studies that showed higher post-operative VTE rates similar to Western populations. In addition, patients who were administered chemoprophylaxis did not have a statistically significant difference in incidence of VTE although it did show a correlation with higher post-operative outcome scores which may indicate better function. This was seen in functional outcome scores such as post op 6 months SF36 (PCS), post op 12 months SF36 (PCS), post op 12 months SF36 (MCS), post op 24 months SF36 (MCS) and post op 24 months WOMAC.

\section{Introduction}

There are over 1 million total hip and total knee replacement procedures performed each year in the United States alone [1]. Demand for hip and knee replacements is rising annually, and growth is expected to be substantial in the years to come due to various reasons including higher rates of diagnosis and treatment of advanced arthritis, as well as increasing demand for improved mobility and quality of life [1]. Venous thromboembolic events (VTE), including deep vein thrombosis (DVT) and pulmonary embolism (PE), are amongst one of the leading causes of morbidity and mortality associated with total knee arthroplasty (TKA) surgeries. $40-50 \%$ of patients with untreated symptomatic DVT will develop a PE within 3 months and $10 \%$ of patients with symptomatic PE die within an hour of onset [2]. In Caucasians, the DVT incidence following total knee arthroplasty (TKA) is reported at $41-85 \%$ and the incidence of pulmonary embolism (PE) of 1.5-10\% [3]. The incidences of DVT reported in Asia following various orthopaedic procedures vary and have been reported to be as high as 53.3\% [4-6]. In our local context, the reported rate of DVT after TKA is $14 \%$ [7].

The efficacy of chemical and mechanical thromboprophylaxis in preventing VTEs in this high-risk group of patients has been well demonstrated. The American Academy of Orthopaedic Surgeons (AAOS) recommends the use of pharmacologic agents and/or mechanical compressive devices for the prevention of venous thromboembolism in patients undergoing elective hip or knee arthroplasty, and who are not at elevated risk beyond that of the surgery itself for venous thromboembolism or bleeding [8]. Similarly, the American College of Chest Physicians (ACCP) states that in patients undergoing TKA, post-surgical chemical thromboprophylaxis with low-molecular-weight heparin (LMWH) or factor Xa inhibitors is recommended for a minimum of 10 to 14 days to reduce the risk and incidence of symptomatic deep vein thrombosis (DVT) and pulmonary embolism (PE) [9].

Despite this, chemoprophylaxis is generally underpracticed in the Asian context [10], owing to concerns of bleeding, slow wound healing and prolonged wound drainage $[11,12]$. In a recent paper, the bleeding from
LMWH (enoxaparin) use after TKA in an Asian population was shown to be as high as $20 \%$ [13]. This reluctance to practise postoperative chemoprophylaxis is reinforced by the belief that the incidence of VTEs has been reported to be lower in the Asian population as compared to the Caucasian population [10]. This raises a debate about whether routine postoperative chemoprophylaxis is required in Asian patients [14].

The primary aim of this study is to determine the incidence of postoperative VTE following TKA in our local population with and without additional chemoprophylaxis in addition to routine mechanical prophylaxis in all patients. The secondary aims are to assess possible risk factors associated with VTE and to compare the 2 year functional outcomes of patients with and without postoperative VTE. We hypothesize that the rate of VTE in TKA patients is comparable to that of Western populations, and that there would be no differences in terms of functional outcomes between patients with and without postoperative VTE.

\section{Materials and methods}

We conducted a retrospective study of all patients who underwent elective TKA from 1st January 2004 to 31st December 2014 at an academic tertiary hospital for osteoarthritis of the knee. Ethics approval was obtained from the Institutional Review Board. Electronic and paper records were reviewed. The data was collected from case notes review, anaesthetic assessment chart reviews and electronic notes. Inclusion criteria were all patients who had underwent elective TKA during the specific time period. Patients who had total knee arthroplasty due to fractures, had unicompartmental knee arthroplasty and who were pre-operatively non ambulatory or minimally ambulatory, were excluded from the study.

\section{Post-operative mobilisation and thromboprophylaxis protocol}

Patients were allowed to stand on the first postoperative day and progressed to full-weight bearing activity with walking aids as tolerated. Each patient was provided with mechanical prophylaxis immediately post operation, which involved the use of both intermittent pneumatic 
compression pumps (ArjoHuntleigh Flowtron ${ }^{\circ}$ Excel DVT pump system) and thromboembolic deterrent open toe knee length compression stockings (T.E.D ${ }^{\text {tu }}$ Knee Length Anti- Embolism Stockings). Additional chemical thromboprophylaxis was administered, subject to the preference of the surgeon and the patient's risk profile. Mechanical prophylaxis was continued until patients were able to ambulate confidently with walking aids for two physiotherapy sessions on the same day. All patients underwent a DUS of both lower limbs within five days after their operation to detect DVT as part of a hospital wide protocol.

\section{Data collection}

Patient demographics including age, gender, significant co-morbidities, and pre-operative mobility status were recorded. Thrombotic risk factors such as hypertension, ischemic heart disease, Diabetes Mellitus, smoking habits, congestive heart failure, previous lower limb VTE and cancer were assessed.

The absence or presence of chemoprophylaxis use, in the form of subcutaneous low molecular weight heparin, was recorded for these patients, together with the presence or absence of VTE. The use of other anti-thrombotic agents postoperatively such as anti-platelet therapy was recorded The presence of DVT was defined as the lack of compressibility and impedance of normal blood in the affected veins, as seen on DUS, with the trifurcation point of the popliteal vein used as the demarcation between proximal and distal deep vein thrombosis. Patients who had symptoms suggestive of PE underwent computed tomography pulmonary angiogram (CTPA) for confirmation of $\mathrm{PE}$, and the presence of symptomatic PE was also documented. Patients were followed up for three months to monitor for any recurrence of VTE.

All patients were followed up with outcome scores for 2 years post operation. Western Ontario and McMaster Universities Osteoarthritis Index [WOMAC] pain), and Short Form-36 (SF-36). Patient outcomes were recorded at six months, twelve months and twenty four months after surgery. These scores enable physicans to have an objective method of assessing the patient's function pre operation and postoperation, and can also be used to assess the patient's general wellbeing.. The WOMAC consists of 24 items divided into 3 subscales - Pain (WOMAC 1), Stiffness (WOMAC 2) and Physical Function (WOMAC 3). The 3 WOMAC subscales were normalized to 0-100 scales to correct for differences in scale range by using the methods recommended by Bellamy. The higher the score, the better the outcomes [15]. With regards to SF-36, the SF-36 is a 36-item patient-reported survey of patient health and reflects quality of life. It consists of eight scaled scores, which are the weighted sums of the questions in their section.
Each scale was transformed into a $0-100$ scale on the assumption that each question carries equal weight. The higher the score, the less disability; a score of zero is equivalent to maximum disability and a score of 100 is equivalent to no disability. The eight sections are: vitality (SFVI), physical functioning (SFPF), bodily pain (SFBP), general health perceptions (SFGHP), physical role functioning (SFPRF), emotional role functioning (SFERF), social role functioning (SFSF) and mental health (SFMH) [16].

Patients who underwent therapeutic anti-coagulation therapy for DVT and/or PE were followed up for a minimum of 3 months to document any bleeding complications from anti-coagulation therapy.

\section{Statistical analysis}

Univariate analyses were performed to describe the presence of DVT in relation to the demographic variables, comorbidities and surgical factors. Mann-Whitney U test was used for numerical prognostic factors, and chi-square test was used for categorical factors. As we noted differences in the age and sex of the patients, and the presence of cancer and previous VTE have been previously shown to demonstrate an increased risk in developing VTE, we adjusted our odds ratios for these four factors.

This was followed by multivariate logistic regression, performed using the variables identified as significant from the univariate analysis. A two-sided $p$-value of $<$ 0.05 was used to select for these variables to be included in in multivariate logistic regression. Subsequent analysis also took a $p$-value of $<0.05$ as unlikely to be due to chance. The logistic regression method used was Forward: Conditional, with an entry probability of 0.05 and a removal probability of 0.10 .

Univariate analysis was also done to describe the type of prophylaxis in relation to functional outcome scores such as the post-op 6 month scores, post-op 12 months scores and post op 24 months scores.

All analysis was performed using IBM Statistical Package for Social Sciences (SPSS) Version 22.

\section{Results}

Using the above-mentioned criteria, 2978 patients were included in this 10-year study (2009 Chinese, 360 Malays, 390 Indians, 35 others). There were 725 men and 2073 women, with a mean age of 65.86 . Mean body mass index was $27.85 \mathrm{~kg} / \mathrm{m} 2$ (range, 24 to $31.5 \mathrm{~kg} / \mathrm{m} 2$ ). This is illustrated in Table 1. All patients received mechanical thromboprophylaxis.

Two thousand two hundred fifty-eight $(80.7 \%)$ patients did not receive additional chemoprophylaxis, while the remaining 540 (19.3\%) received chemoprophylaxis along with mechanical thromboprophylaxis. All patients who received chemoprophylaxis were administered the enoxaparin 


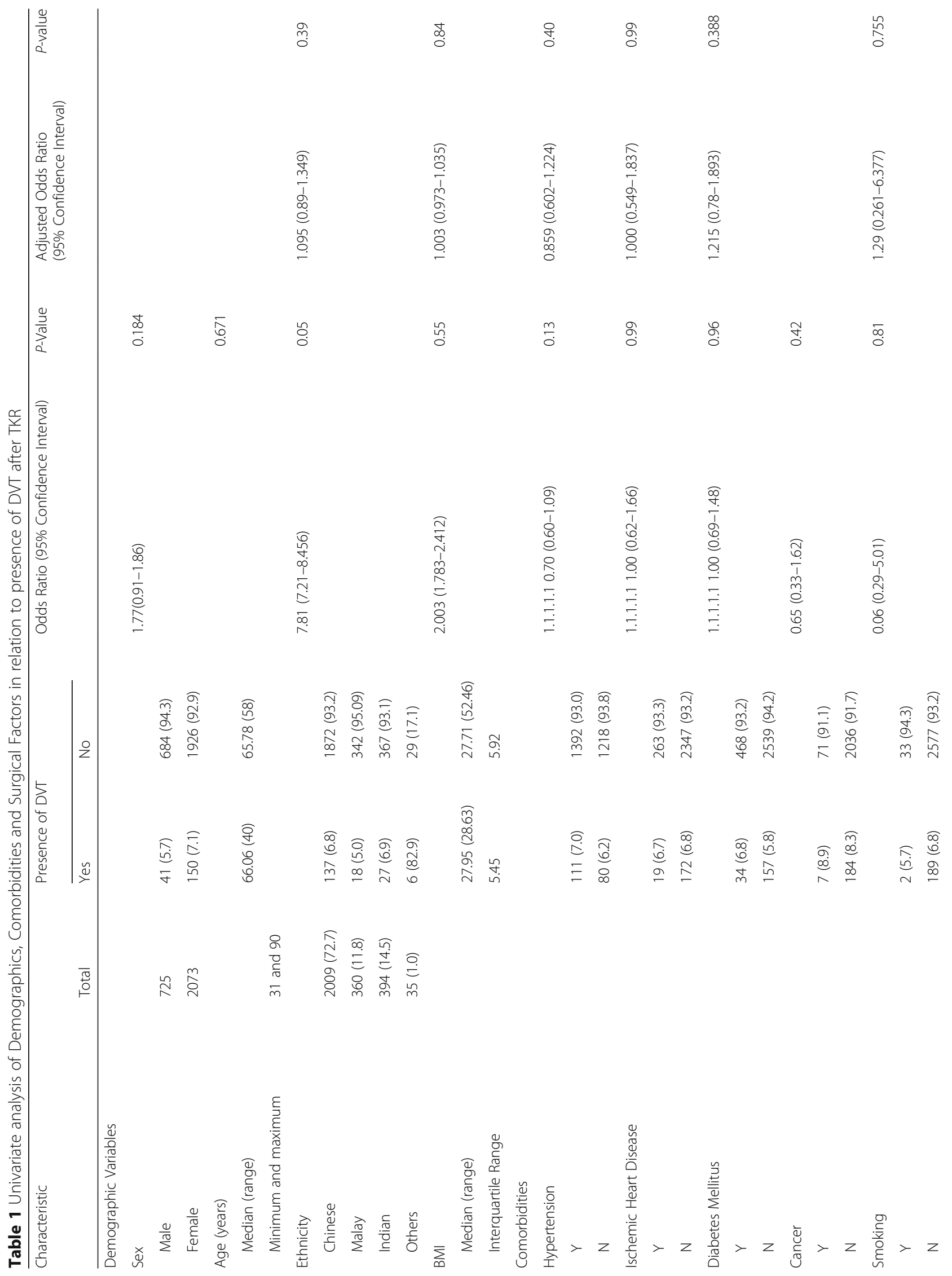




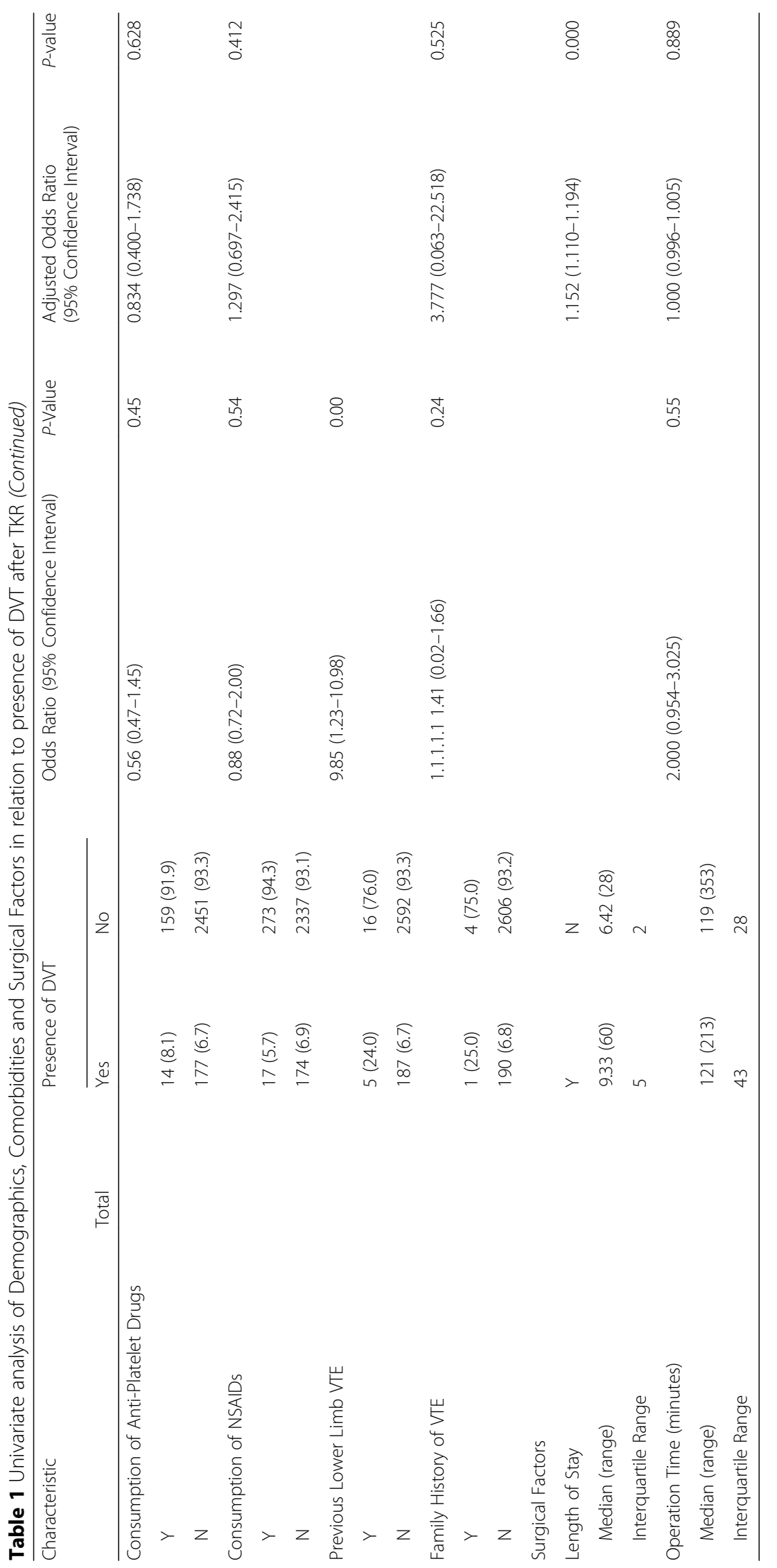


until they were ambulating, with a median administration duration of 6 days (minimum 2 days, maximum 30 days).

\section{Overall rates of VTEs}

Two thousand nine hundred seventy-eight patients had DUS of the lower limbs with 134 diagnosed with DVT with an incidence of $4.5 \%$. There were $26(19.4 \%)$ proximal DVTs and 108 (80.6\%) distal DVTs. In addition, there were 6 PEs diagnosed on computer tomography of the chest. At 3 months of follow up, no additional VTE occurred.

Table 2 shows the sites of thrombi identified by the duplex Doppler ultrasound. All DVTs with accompanying PE were proximal.

Table 3 shows the method of treatment for the identified DVTs. Almost 50\% were simply monitored while the other $50 \%$ were anticoagulated. Treatment of the DVTs was heterogeneous - in some cases, haematologists were consulted while in some cases, they were not. All proximal DVTs were anticoagulated. Out of the distal DVTs, only posterior tibial veins were not anticoagulated.

\section{Subgroup analysis: comparison between patients with and without chemoprophylaxis}

One hundred two out of 2200 patients (4.6\%) without chemoprophylaxis developed DVT as compared to 32 out of 540 patients $(5.9 \%)$ with chemoprophylaxis, which was not statistically significant $(p=0.13) .19(0.8 \%)$ proximal and $83(3.8 \%)$ distal DVT developed in the patient group without chemoprophylaxis while 4 (0.7\%) proximal and 28 (5.2\%) distal DVT developed in the patient group with $(p=0.62)$. Comparison of the incidence of PEs between the two groups, revealed a similar incidence with 5 out of 2200 patients (0.2\%) without

Table 2 Sites of Lower Limb Venous Thromboses by Number and Percentage

\begin{tabular}{ll}
\hline Site & Number of Patients \\
\hline Knees with no thrombi & 2978 \\
Knees with DVT & $134(100 \%)$ \\
Proximal DVT & $26(19.4 \%)$ \\
Superficial Femoral Vein & 6 \\
Popliteal Vein & 20 \\
Distal DVT & $108(80.6 \%)$ \\
Soleal & 14 \\
Peroneal & 64 \\
Posterior Tibial & 30 \\
Superfical Venous Thromboses & \\
Calf (Intramuscular) & 27 \\
Calf (Superficial) & 2 \\
Great Saphenous & 15 \\
\hline
\end{tabular}

Table 3 Type of Treatment for Patients with Lower Limb Venous Thromboses

\begin{tabular}{ll}
\hline Type of DVT Treatment & Number of Patients \\
\hline Monitor & 66 \\
Anticoagulation & 67 \\
\hline
\end{tabular}

chemoprophylaxis developing $\mathrm{PE}$ as compared to 1 out of 540 patients $(0.2 \%)$ with chemoprophylaxis $(p=0.87)$. 2 patients had bleeding complications - one from the group with chemoprophylaxis and one from the group without chemoprophylaxis.

Table 4 shows the comparison of the functional outcome scores with and without chemical prophylaxis. Post op 6 months SF36 (MCS), post op 12 months SF36 (MCS), post op24 months SF36 (MCS), and post op 24 months WOMAC are statistically significant.

Table 5 shows the results of multivariate analysis using demographics, comorbidities, and surgical factors found to be significant on univariate analysis. For multivariate analysis, length of stay and previous lower limb VTE are significant.

Table 6 shows the results of multivariate analysis using the postoperative outcome scores which were found to be significant on univariate analysis. For multivariate analysis, post op 6 months SF36 (PCS) and post op 24 months SF36 (MCS) are significant. The analysis showed that the type of thromboprophylaxis was independently associated with post op 6 months SF36 (PCS), post op and post op 24 months SF36 (MCS).

\section{Discussion}

We showed that the incidence of DVTs in a multi-ethnic Asian population post elective total knee arthroplasty with mechanical prophylaxis is $4.6 \%$. The use of mechanical and chemical prophylaxis did not lower the risk of developing DVT (incidence of 5.9\%). Comparisons between the patient groups with and without chemoprophylaxis revealed that all DVT that developed with the use of chemoprophylaxis were proximal DVTs, suggesting that chemoprophylaxis could possibly have reduced the incidence of distal DVT more effectively than that of proximal DVT. This observation could also possibly explicate the relative ineffectiveness of chemoprophylaxis in reducing the incidence of pulmonary embolism, since pulmonary emboli were more strongly correlated with proximal DVT as found in our study. This observation has been noted in a few previous studies of total knee arthroplasty, where chemoprophylaxis has been found to be ineffective in reducing the incidence of PE $[17,18]$.

Patients who undergo total knee arthroplasty have been identified to have a high risk for VTE, leading to recommendations in international guidelines to use a 
Table 4 Functional Outcome Scores of Patients with and without Chemoprophylaxis

\begin{tabular}{llll}
\hline Type of Outcome & With chemical prophylaxis & Without chemical prophylaxis & $P$-value \\
\hline Post-Op 6 months SF-36 PCS & 85.6 & 82.5 & 0.682 \\
Post-Op 6 months SF-36 MCS & 78.6 & 94.3 & 0.00 \\
Post-Op 6 months WOMAC & 82.6 & 83.0 & 74.4 \\
Post-Op 12 months SF-36 PCS & 76.0 & 68.8 & 0.914 \\
Post-Op 12 months SF-36 MCS & 82.4 & 72.3 & 0.118 \\
Post-Op 12 months WOMAC & 70.8 & 55.8 & 0.00 \\
Post-Op 24 months SF-36 PCS & 55.3 & 54.6 & 0.567 \\
Post-Op 24 months SF-36 MCS & 61.7 & 56.5 & 0.573 \\
Post-Op 24 months WOMAC & 51.9 & & 0.006 \\
\hline
\end{tabular}

combination of mechanical and chemical thromboprophylaxis before and after the procedure $[19,20]$.

Pharmacological thromboprophylaxis has been recommended to reduce the prevalence of postoperative DVT on the assumption that it will reduce the prevalence of PE, mortality, and thrombophlebitic syndrome [21, 22]. However, pharmacological thromboprophylaxis itself carries several risks such as the risk of bleeding, complications of blood loss, transfusion, transfusion-related transmission of disease, wound-healing problems, hematoma, slowed rehabilitation, wound drainage, and infection [23]. The practice of routine postoperative chemical thromboprophylaxis to prevent VTE has mainly been based on Western literature thus far, and the recent acknowledgement of studies which show a lower incidence of thromboembolism in Asia [24] has led to questions regarding the need for routine chemoprophylaxis for patients undergoing total knee arthroplasty.

Recent studies done in Asian populations however have shown otherwise, with the incidence of DVT being shown to be comparable in both Caucasian and Asian populations [25-27]. Treatment should ideally be individualized to the patient's risk profile, raising the question of Asian populations possibly following different guidelines for thromboprophylaxis after TKA. Current guidelines recommend differing modalities of

Table 5 Multivariate analysis using demographics, comorbidities and surgical factors found to be significant on univariate analysis

\begin{tabular}{lll}
\hline Statement & $\begin{array}{l}\text { Odds Ratio } \\
\text { (95\% Confidence interval) }\end{array}$ & $P$-value \\
\hline Ethnicity & 0.184 & 0.089 \\
$\quad$ Chinese & 0.400 & \\
Malay & 0.275 & \\
Indian & 0.354 & \\
Others & $1.134(1.101-1.168)$ & 0.00 \\
Length of Stay & $3.963(1.327-11.831)$ & 0.014 \\
\hline
\end{tabular}

thromboprophylaxis depending on the patient's risk factors. The AAOS recommends that patients who have had a previous VTE should receive the combined modalities of pharmacologic prophylaxis and mechanical prophylaxis, such as thromboembolic deterrent (TED) stockings or intermittent pneumatic compressive devices (IPCD). In patients who have a known bleeding disorder (e.g., hemophilia) and/or active liver disease, only mechanical compressive devices should be used for preventing VTE [8]. This is identical to the ACCP which also advocates combined modalities for normal patients and solely mechanical prophylaxis or no prophylaxis for patients with an increased risk of bleeding [9]. Significantly, in a multicenter randomized controlled trial comparing IPCD against enoxaparin, Colwell et al. showed that IPCD was just as effective as enoxaparin in preventing proximal and distal DVT and PE events in hip arthroplasty patients, but resulted in a much lower bleeding risk (1.3\% IPCD vs $4.3 \%$ LMWH) [28]. This adds to the argument that patients with a decreased risk of VTE should not undergo chemoprophylaxis but use only mechanical prophylaxis, thereby sparing them the adverse risk of bleeding. However, it is still unclear whether combined modalities of chemical and mechanical prophylaxis are better than either chemoprophylaxis or mechanical prophylaxis alone in overall functional outcomes. A study in 2013 found that there were no significant differences in the patient-reported quality of life outcomes and therapist-reported knee range of motion between patients who had developed DVT and those who had not [17]. Our study showed that the type

Table 6 Multivariate Analysis Using Postoperative Outcome Scores Found to be Significant on Univariate Analysis

\begin{tabular}{lll}
\hline Statement & $\begin{array}{l}\text { Odds Ratio (95\% Confidence } \\
\text { interval) }\end{array}$ & $p$-value \\
\hline post op 6 months SF36 (MCS) & $1.182(0.566-1.571)$ & 0.032 \\
post op 12 months SF36 (MCS) & $0.971(0.569-1.574)$ & 0.132 \\
post op 24 months SF36 (MCS) & $1.063(0.573-1.578)$ & 0.014 \\
post op 12 months WOMAC & $1.342(0.888-1.900)$ & 0.21 \\
\hline
\end{tabular}


of thromboprophylaxis was independently associated with post op 6 months SF36 (PCS) and post op 24 months SF36 (MCS). Anticoagulation has been associated with decreased risk of acute myocardial infarction and stroke [29]. Given our patient demographics, it is possible this could have played a role in higher patient outcome scores despite no significant difference in VTE incidence. Further studies will have to be done to explore this finding.

Our incidence of VTE is low compared to that of Caucasian populations. In a review of the literature, there is only a single study on a Caucasian population which revealed similar a similar incidence of DVT -Gelfer et al. showed an incidence of DVT of $6.6 \%$ in a THA and TKA population that was treated only by mechanical prophylaxis therapy coupled with aspirin [30]. An explanation for this difference between the Asian and Western populations is most likely due to the prevalence of prothrombotic factors in Caucasians which cause them to have a higher risk of developing VTEs as compared to Asians, regardless of the type of surgery performed [31]. In fact, the low probability of Asian populations having postoperative DVT and PE as compared to Western populations was first highlighted by Tinckler et al. in 1964 [32]. There are some data that support genetic differences as a partial cause of a lower risk of VTE in Asians. The most common of the known genetic mutations is a gene known as factor V Leiden [33] which increases DVT risk by about 7 times in heterozygotes, and about 80 times in homozygotes. It has been shown to be found in approximately $5 \%$ of Caucasians, but is less common in Africans and rare in Asians [34]. Another genetic trait that predisposes to DVT and PE is the prothrombin promoter G20210A mutation. This is found in 4 to $6 \%$ of Caucasians and enhances transcription of the prothrombin gene, yielding higher prothrombin levels and consequently easier generation of thrombin, the key enzyme in blood clotting [35].

Last but not least, our study noted an apparent correlation between proximal DVT as well as bilateral DVT and the development of PE. All the PEs that had an accompanying DVT in the study had proximal DVT. Li et al. had identified a similar relationship in her study which showed the risk of developing a silent PE in patients with proximal DVT [36]. Larger scale studies would be beneficial in further investigating this possible relationship. Strengths of our study are that we have a large cohort of patients in a 10 year follow up period with no loss to follow up in the public sector. This is due to our National Electronic Healthcare Registry in Singapore which combines patient information in all our public hospitals. However as it does not include the private hospitals, there could have been loss to follow up to private institutions during the study period. Care was also standardized across the study as the methods were part of the hospital protocol. It is also one of the first studies to show a difference in functional outcomes scores (SF-36) between the type of thromboprophylaxis for total knee arthroplasty. A limitation of our study is that we employed the usage of Doppler Ultrasound, which is operator dependent. Thus we could have missed out on certain DVTs that could have been picked up by venograms.

In Asian patients on thromboprophylaxis post-TKA, there is no significant difference in incidence of DVT between patients on chemoprophylaxis to those without. Mechanical postoperative thromboprophylaxis may be adequate in post-TKA DVT prevention, in our local context. A randomized controlled trial in Asians should be done to assess if the addition of chemoprophylaxis on top of mechanical thromboprophylaxis might lead to more harm than good.

\section{Conclusion}

In one of the largest Asian studies specifically investigating the incidence of DVT after TKA, we found that the incidence is low at $4.5 \%$. The study institution's policy for VTE prevention included routine mechanical thromboprophylaxis and duplex ultrasound. With this policy, VTE rates that are clinically relevant were very low.

This is in contrast to recent studies that showed higher post-operative VTE rates similar to Western populations.. In addition, patients who were administered chemoprophylaxis did not have a statistically significant difference in incidence of VTE although it did show a correlation with higher post-operative outcome scores indicating better function.

\section{Abbreviations}

BMI: Body Mass Index; Cl: Confidence Interval; DVT: Deep Venous

Thrombosis; OR: Odd Ratio; PE: Pulmonary Embolism; SF-36: Short Form-36; TKA: Total Knee Arthroplasty; VTE: Venous Thromboembolism;

WOMAC: Western Ontario and McMaster Universities Osteoarthritis Index

\section{Acknowledgements}

Not applicable.

\section{Funding}

Not applicable.

\section{Availability of data and materials}

The datasets used and/or analysed during the current study are available from the corresponding author on reasonable request.

\section{Authors' contributions \\ LLLM authored the manuscript and gathered the data. LJLM analysed and interpreted the data. ESY and WKL supervised the work and edited the article. All authors read and approved the final manuscript.}

Ethics approval and consent to participate

Independent ethics committee and hospital-based institutional review board approvals were obtained, as necessary, for the registry protocol.

Consent for publication

Not applicable. 


\section{Competing interests}

The authors declare that they have no competing interests.

\section{Publisher's Note}

Springer Nature remains neutral with regard to jurisdictional claims in published maps and institutional affiliations.

\section{Author details}

'Department of Orthopaedic Surgery, Singapore General Hospital, 20 College Road, Academia, Level 4, Singapore 169865, Singapore. ${ }^{2}$ Department of Haematology, Tan Tock Seng Hospital, Singapore, Singapore. ${ }^{3}$ Department of Orthopaedic Surgery, Sengkang General Hospital, Singapore, Singapore. ${ }^{4}$ Department of Hematology-Oncology, National University Cancer Institute, National University Hospital, Singapore, Singapore.

Received: 6 February 2019 Accepted: 9 May 2019

Published online: 20 June 2019

\section{References}

1. Steiner C, Andrews R, Barrett M, Weiss A. HCUP projections: mobility/ orthopedic procedures 2003 to 2012. HCUP projections report\# 2012-03 2012. US Agency for Healthcare Research and Quality.

2. Geerts WH, Bergqvist D, Pineo GF, Heit JA, Samama CM, Lassen MR, Colwell CW. Prevention of venous thromboembolism: American College of Chest Physicians evidence-based clinical practice guidelines. Chest. 2008;133(6): 381-453.

3. Cohen AT. Asia-Pacific thrombosis advisory board consensus paper on prevention of venous thromboembolism after major orthopaedic surgery. Thromb Haemost. 2010;104(05):919-30.

4. Atichartakarn V, Pathepchotiwong K, Keorochana S, Eurvilaichit C. Deep vein thrombosis after hip surgery among Thai. Arch Intern Med. 1988;148(6): 1349-53.

5. Kim YH, Suh JS. Low incidence of deep-vein thrombosis after cementless total hip. J Bone Joint Surg Am. 1988;70:878-82.

6. Mok CK, Hoaglund FT, Rogoff SM, Chow SP, Yau AC. The pattern of deepvein thrombosis and clinical course of a group of Hong Kong Chinese patients following hip surgery for fracture of the proximal femur. Clin Orthop Relat Res. 1980;(147):115-20.

7. Lee LH. Clinical update on deep vein thrombosis in Singapore. Ann Acad Med Singap. 2002;31(2):248-52.

8. Mont MA, Jacobs JJ, Boggio LN, Bozic KJ, Della Valle CJ, Goodman SB, Lewis CG, Yates AJ Jr, Watters WC III, Turkelson CM, Wies JL. Preventing venous thromboembolic disease in patients undergoing elective hip and knee arthroplasty. J Am Acad Orthop Surg. 2011;19(12):768-76.

9. Falck-Ytter Y, Francis CW, Johanson NA, Curley C, Dahl OE, Schulman S, Ortel TL, Pauker SG, Colwell CW Jr. Prevention of VTE in orthopedic surgery patients: antithrombotic therapy and prevention of thrombosis: American College of Chest Physicians evidence-based clinical practice guidelines. Chest. 2012;141(2):e278S-325S.

10. Dhillon KS, Askander A, Doraisamy S. Postoperative deep-vein thrombosis in Asian patients is not a rarity: a prospective study of 88 patients with no prophylaxis. J Bone Joint Surg Br. 1996;78(3):427-30.

11. Patel VP, Walsh M, Sehgal B, Preston C, DeWal H, Di Cesare PE. Factors associated with prolonged wound drainage after primary total hip and knee arthroplasty. JBJS. 2007:89(1):33-8.

12. Lyder CH. Pressure ulcer prevention and management. Jama. 2003;289(2): 223-6.

13. Fuji T, Nakamura M, Takeuchi M. Darexaban for the prevention of venous thromboembolism in Asian patients undergoing orthopedic surgery: results from 2 randomized, placebo-controlled, double-blind studies. Clin Appl Thromb Hemost. 2014;20(2):199-211.

14. Kanchanabat B, Stapanavatr W, Meknavin S, Soorapanth C, Sumanasrethakul C, Kanchanasuttirak P. Systematic review and meta-analysis on the rate of postoperative venous thromboembolism in orthopaedic surgery in Asian patients without thromboprophylaxis. Br J Surg. 2011;98(10):1356-64.

15. Bellamy N. WOMAC Osteoarthritis index: a user's guide. London, Ontario: University of Western Ontario; 1996

16. Gandek B, Ware JE Jr, Aaronson NK, Alonso J, Apolone G, Bjorner J, Brazier J, Bullinger M, Fukuhara S, Kaasa S, Leplège A. Tests of data quality, scaling assumptions, and reliability of the SF-36 in eleven countries: results from the IQOLA Project. J. Clin. Epidemiol. 1998;51(11):1149-58.
17. Zhou Z, Yew AK, Chin PL, Lo NN, Yeo SJ, Chia SL. Total knee arthroplasty complicated by distal deep venous thromboembolism: does it affect the functional outcome? Proceedings of Singapore Healthcare. 2013;22(4):262-6.

18. Hill J, Treasure T. Reducing the risk of venous thromboembolism (deep vein thrombosis and pulmonary embolism) in patients admitted to hospital: summary of the NICE guideline. Heart. 2010;96(11):879-82.

19. Kearon C, Akl EA, Comerota AJ, Prandoni P, Bounameaux H, Goldhaber SZ, Nelson ME, Wells PS, Gould MK, Dentali F, Crowther M. Antithrombotic therapy for VTE disease: antithrombotic therapy and prevention of thrombosis: American College of Chest Physicians evidence-based clinical practice guidelines. Chest. 2012 1;141(2):e419S-96S.

20. Eikelboom JW, Karthikeyan G, Fagel N, Hirsh J. American Association of Orthopedic Surgeons and American College of Chest Physicians guidelines for venous thromboembolism prevention in hip and knee arthroplasty differ: what are the implications for clinicians and patients? Chest. 2009;135(2):513-20.

21. Sharfman ZT, Campbell JC, Mirocha JM, Spitzer Al. Balancing thromboprophylaxis and bleeding in total joint arthroplasty: impact of eliminating enoxaparin and predonation and implementing pneumatic compression and tranexamic acid. J arthroplasty. 2016;31(6):1307-12.

22. Cusick LA, Beverland DE. The incidence of fatal pulmonary embolism after primary hip and knee replacement in a consecutive series of 4253 patients. J Bone Joint Surg Br. 2009:91(5):645-8.

23. Sharrock NE, Della Valle AG, Go G, Lyman S, Salvati EA. Potent anticoagulants are associated with a higher all-cause mortality rate after hip and knee arthroplasty. Clin Orthop Relat Res. 2008;466(3):714-21.

24. Wong KL, Daguman $\mathrm{R}$, Lim KH, Shen L, Lingaraj K. Incidence of deep vein thrombosis following total hip arthroplasty: a Doppler ultrasonographic study. J Orthop Surg. 2011:19(1):50-3.

25. Wang CJ, Wang JW, Chen LM, Chen HS, Yang BY, Cheng SM. Deep vein thrombosis after total knee arthroplasty. J Formos Med Assoc. 2000;99(11): 848-53.

26. Ko PS, Chan WF, Siu TH, Khoo J, Wu WC, Lam JJ. Deep venous thrombosis after total hip or knee arthroplasty in a "low-risk" Chinese population. J Arthroplast. 2003;18(2):174-9.

27. Piovella F, Wang CJ, Lu H, Lee K, Lee LH, Lee WC, Turpie AG, Gallus AS, Planès A, Passera R, Rouillon A. Deep-vein thrombosis rates after major orthopedic surgery in Asia. An epidemiological study based on postoperative screening with centrally adjudicated bilateral venography. J Thromb Haemost. 2005;3(12):2664-70.

28. Colwell CW Jr, Froimson MI, Mont MA, Ritter MA, Trousdale RT, Buehler KC, Spitzer A, Donaldson TK, Padgett DE. Thrombosis prevention after total hip arthroplasty: a prospective, randomized trial comparing a mobile compression device with low-molecular-weight heparin. JBJS. 2010;92(3):527-35.

29. Clayville LR, Anderson KV, Miller SA, Onge EL. New options in anticoagulation for the prevention of venous thromboembolism and stroke. Pharmacy and Therapeutics. 2011;36(2):86.

30. Gelfer $Y$, Tavor H, Oron A, Peer A, Halperin N, Robinson D. Deep vein thrombosis prevention in joint arthroplasties: continuous enhanced circulation therapy vs low molecular weight heparin. J arthroplasty. 2006:21(2):206-14

31. White $\mathrm{RH}$, Keenan CR. Effects of race and ethnicity on the incidence of venous thromboembolism. Thromb Res. 2009;123:S11-7.

32. Tinckler LF. Absence of pulmonary embolism in asians? Br Med J. 1964; 1(5381):502.

33. Klatsky AL, Armstrong MA, Poggi J. Risk of pulmonary embolism and/or deep venous thrombosis in Asian-Americans. Am J Cardiol. 2000;85(11):1334-7.

34. De Stefano V, Martinelli I, Mannucci PM, Paciaroni K, Chiusolo P, Casorelli I, Rossi $\mathrm{E}$, Leone $\mathrm{G}$. The risk of recurrent deep venous thrombosis among heterozygous carriers of both factor $V$ Leiden and the G20210A prothrombin mutation. N Engl J Med. 1999;341(11):801-6.

35. Hessner MJ, Luhm RA, Pearson SL, Endean DJ, Friedman KD, Montgomery RR. Prevalence of prothrombin G20210A, factor V G1691A (Leiden), and methylenetetrahydrofolate reductase (MTHFR) C677T in seven different populations determined by multiplex allele-specific PCR. Thromb Haemost. 1999:81(05):733-8

36. Li F, Wang $X$, Huang W, Ren W, Cheng J, Zhang M, Zhao Y. Risk factors associated with the occurrence of silent pulmonary embolism in patients with deep venous thrombosis of the lower limb. Phlebology. 2014;29(7):442-6. 